University of Nebraska - Lincoln

DigitalCommons@University of Nebraska - Lincoln

6-3-2002

\title{
Piezoresponse force microscopy for polarity imaging of GaN
}

\section{B.J. Rodriguez}

North Carolina State University, Raleigh, brian.rodriguez@ucd.ie

Alexei Gruverman

University of Nebraska-Lincoln, agruverman2@unl.edu

A. I. Kingon

North Carolina State University, angus_kingon@brown.edu

R. J. Nemanich

North Carolina State University, Box 8202, Raleigh, North Carolina

Follow this and additional works at: https://digitalcommons.unl.edu/physicsgruverman

Part of the Physics Commons

Rodriguez, B.J.; Gruverman, Alexei; Kingon, A. I.; and Nemanich, R. J., "Piezoresponse force microscopy for polarity imaging of GaN" (2002). Alexei Gruverman Publications. 10.

https://digitalcommons.unl.edu/physicsgruverman/10

This Article is brought to you for free and open access by the Research Papers in Physics and Astronomy at DigitalCommons@University of Nebraska - Lincoln. It has been accepted for inclusion in Alexei Gruverman Publications by an authorized administrator of DigitalCommons@University of Nebraska - Lincoln. 


\title{
Piezoresponse force microscopy for polarity imaging of GaN
}

\author{
B. J. Rodriguez, A. Gruverman, A. I. Kingon, and R. J. Nemanich ${ }^{\text {a) }}$ \\ Department of Physics and Department of Materials Science and Engineering, North Carolina State \\ University, Box 8202, Raleigh, North Carolina, 27695-8202 \\ O. Ambacher \\ Walter Schottky Institute, Technical University Munich, Am Coulombwall, 85748 Garching, Germany
}

(Received 11 February 2002; accepted for publication 9 April 2002)

The polarity distribution of $\mathrm{GaN}$ based lateral polarity heterostructures is investigated by piezoresponse force microscopy (PFM). Simultaneous imaging of surface morphology, as well as the phase and magnitude of the piezoelectric response, is performed by PFM on a GaN film with patterned polarities on a $c-\mathrm{Al}_{2} \mathrm{O}_{3}$ substrate. We demonstrate that the polarity distribution of $\mathrm{GaN}$ based lateral polarity heterostructures can be deduced from the phase image of the piezoresponse with nanometer scale spatial resolution. (C) 2002 American Institute of Physics.

[DOI: $10.1063 / 1.1483117$ ]

The piezoelectric character of III-nitride semiconductors yields a novel degree of freedom in designing devices for modern micro- and nanoelectronic applications. ${ }^{1,2}$ As such, measurement of the piezoelectric properties of III-nitrides with high spatial resolution is of importance for the design and performance of $\mathrm{GaN}$ based electronic and optoelectronic devices. Moreover, investigation of the piezoelectric behavior of nitride thin films and heterostructures at the nanoscale level is of considerable interest for determining how interfaces, dislocations, stacking faults, and inversion domain boundaries can affect device performance.

In their wurtzite phase, III-nitrides are piezoelectric as well as pyroelectric materials having piezoelectric constants $d_{i j}$, which are about one order of magnitude higher than in other III-V compound semiconductors. ${ }^{2}$ The spontaneous polarizations for $\mathrm{GaN}$ and $\mathrm{AlN}$ are $P_{\mathrm{SP}}=-0.034$, and -0.090 $\mathrm{C} / \mathrm{m}^{2}$, respectively. ${ }^{2}$ In $\mathrm{GaN}$ the [0001]-axis points from the $\mathrm{Ga}$ atom to the nearest neighbor $\mathrm{N}$ atom (cation to anion) along the crystallographic $c$-axis, and from the $\mathrm{N}$ face to the Ga face (since Ga atoms will be on the top position of the $\{0001\}$ bilayer). The orientation of spontaneous polarization is defined by convention such that the positive direction is along the crystallographic [0001] axis. Since the sign of the spontaneous polarization is predicted to be negative, the orientation always points from the $\mathrm{Ga}$ face toward the $\mathrm{N}$ face side of the crystal (in the $[000 \overline{1}]$ direction). ${ }^{1-3}$ In contrast to ferroelectric materials, the orientation of the spontaneous polarization in nitrides cannot be changed by the application of an external electric field.

At the surfaces of relaxed GaN and AlN crystals, a gradient in the spontaneous polarization induces polarization bound surface charges with densities of $2.12 \times 10^{13}$ and $5.62 \times 10^{13} \mathrm{~cm}^{-2}$, respectively. The sign of the polarization induced charge at each surface is related to the orientation of the polarization and therefore to the polarity of the crystal. For epitaxial layers of GaN with Ga-face polarity, the bound surface charge is negative, whereas for $\mathrm{N}$-face $\mathrm{GaN}$ the bound surface charge is positive. The polarization induced

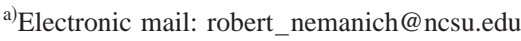

surface charge is balanced by a bound charge of opposite sign at the nitride/substrate interface. ${ }^{3}$

While the predicted spontaneous polarization is independent of strain, piezoelectric polarization is strain-induced. ${ }^{3}$ In general, the total polarization of a nitride layer is the sum of the spontaneous and of the piezoelectric polarizations. As shown by Ambacher et al. ${ }^{1,3}$ piezoelectric and spontaneous polarizations are parallel for tensile strain and are antiparallel for compressive strain.

Recently, scanning probe microscopy (SPM) based techniques were employed to perform high-resolution characterization of the local electronic properties of III-nitrides. ${ }^{4-6}$ In this study, we present another SPM based technique, namely, piezoresponse force microscopy (PFM) to investigate the local electromechanical properties of III-nitrides. PFM offers significant advantage compared to macroscopic techniques $^{7-9}$ in studying the properties of piezoelectric films. In addition to measuring the piezoelectric displacement with sub-Angstrom precision, in the PFM approach, the tip can be rastered to generate 2-dimensional images of the magnitude and sign of the piezoresponse signal with nanoscale lateral resolution. As such, PFM can resolve nanometer variations in the piezoelectric properties of a sample, and is ideally suited for investigation of the piezoelectric properties near inversion domains and other defects. In this paper, we report nanoscale imaging of $\mathrm{GaN}$ heterostructures with patterned polarities on a sapphire substrate using PFM.

The PFM imaging method, which was developed to visualize domain structure in ferroelectric thin films, is described in detail elsewhere. ${ }^{10-16}$ Ideally, with PFM when a modulation voltage is applied to a piezoelectric material, the vertical displacement of the probing tip, which is in mechanical contact with the sample, accurately follows the piezoelectric motion of the sample surface. The voltage $V$ applied across a film of thickness $t$, generates an electric field $E_{3}$ along the $c$-axis, which strains the film by a thickness $\Delta t$. The $d_{33}$ piezoelectric coefficient is then proportional to the applied voltage: $d_{33}=\Delta S_{3} / E_{3}=\Delta t / V$, where $E_{3}=V / t$, and $\Delta S_{3}=\Delta t / t$ is the change of strain along the $c$-axis. Thus, the amplitude of the tip vibration measured by the lock-in tech- 
nique provides information on the piezoelectric strain and coefficient $d_{33}$ can be determined. The polarity distribution of the GaN heterostructure can be determined based on the dependence of the sign of the piezoelectric coefficient on the polarization direction; the oscillation of the film is either in-phase or out-of-phase with the modulation voltage, corresponding to either a Ga- or N-face film, respectively. Therefore, in a PFM phase image, Ga- and $\mathrm{N}$-face polarities will exhibit opposite contrast.

There are two main approaches in PFM: measurements can be performed by applying a voltage between a conducting tip and a bottom electrode, or between deposited top and bottom electrodes. For example, Gruverman et al. ${ }^{11}$ performed measurements of $\mathrm{Pb}\left(\mathrm{Zr}_{x}, \mathrm{Ti}_{1-x}\right) \mathrm{O}_{3}$ (PZT) thin films by applying a voltage between a bottom electrode and a conducting tip used as a moveable electrode. With this configuration, the polarization distribution in PZT thin films were imaged with a lateral resolution of about $10 \mathrm{~nm} .{ }^{11}$ However, the electric field generated by the PFM tip is highly inhomogeneous, which makes quantitative measurements of the field dependent parameters difficult. Alternatively, Christman et al. ${ }^{15}$ made measurements by applying a voltage between deposited top and bottom electrodes and demonstrated a submicron variation of piezoelectric properties in PZT capacitors. With this configuration, a homogeneous electric field is generated throughout the piezoelectric layer and the electrostatic tip-sample interaction is suppressed, although at the expense of lower lateral resolution.

In this study, the first approach was utilized to perform PFM with a TM Microscopes AutoProbe M5, conducting tips, and a dual-phase lock-in amplifier (SR830). ${ }^{16}$ The commercially available conducting cantilevers (force constant $2.1 \mathrm{~N} / \mathrm{m}$ ) had a $1000 \AA$ layer of $0.1 \Omega \mathrm{cm} p$-type diamond over the Si tips and were mounted on alumina squares. To explore the imaging capability of PFM, and the influence of polarity on the amplitude and the phase of the measured piezoresponse, a plasma induced molecular beam epitaxy grown $\mathrm{GaN}$ film with patterned $\mathrm{Ga}$ - and $\mathrm{N}$-face polarities was examined at a modulation frequency of $10 \mathrm{kHz}$ with a driving voltage of $5 \mathrm{~V}$ applied to the tip. The Ga-face GaN (1 $\mu \mathrm{m}$ thick) was grown on an AlN nucleation layer $(10 \mathrm{~nm}$ thick), while the $\mathrm{N}$-face $\mathrm{GaN}$ (1 $\mu \mathrm{m}$ thick) was grown directly on the sapphire substrate. ${ }^{17}$

Figure 1(a) shows a topographic image of the GaN film with alternating concentric squares of $\mathrm{Ga}$ and $\mathrm{N}$ faces (the innermost square is Ga face). In Figs. 1(b) and 1(c), PFM magnitude and PFM phase images, respectively, are displayed. It is interesting to note that the piezoresponse magnitude, which should be independent of polarity, is higher [as denoted by a lighter contrast in Fig. 1(b)] for the N-face $\mathrm{GaN}$. We suggest this results from the better structural quality of the $\mathrm{N}$-face in comparison to $\mathrm{Ga}$-face $\mathrm{GaN}$ (dislocation density is $\approx 8 \times 10^{9}$ and $\approx 1 \times 10^{9} \mathrm{~cm}^{-2}$ for Ga- and N-face $\mathrm{GaN}$, respectively) and the slightly different biaxial compressive strain, which was measured using $\mathrm{x}$-ray diffraction to be $6.35 \times 10^{-3}$ and $5.47 \times 10^{-3}$ for the Ga- and $\mathrm{N}$-face regions, respectively. This strain causes a piezoelectric polarization of $+0.0055 \mathrm{C} / \mathrm{m}^{2}$ for the Ga face and of $-0.0047 \mathrm{C} / \mathrm{m}^{2}$ for the $\mathrm{N}$ face. Accounting for spontaneous polarization $(-0.034$ $\mathrm{C} / \mathrm{m}^{2}$ and $+0.034 \mathrm{C} / \mathrm{m}^{2}$ for $\mathrm{Ga}$ and $\mathrm{N}$ face, respectively), the

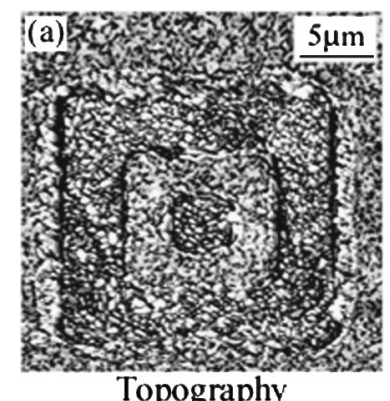

Topography

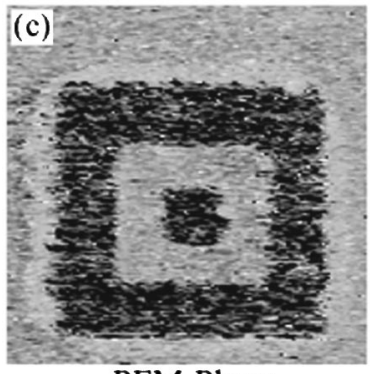

PFM Phase

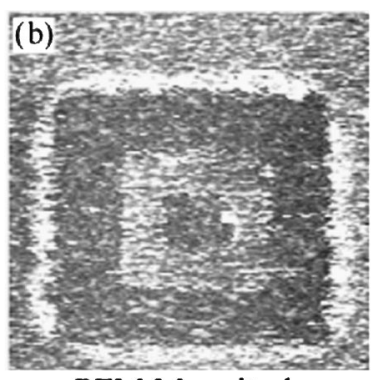

PFM Magnitude
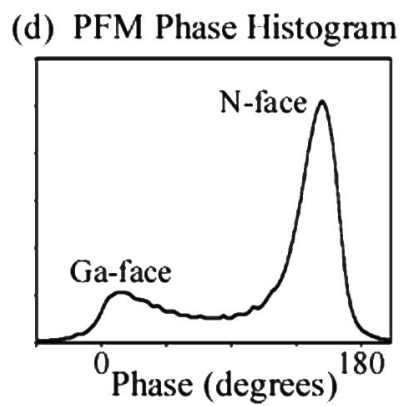

FIG. 1. (a) Topographic, (b) PFM magnitude, and (c) PFM phase images of a GaN-based lateral polarity heterostructure. The innermost $5 \times 5 \mu \mathrm{m}^{2}$ square is the Ga-face region. The $\mathrm{N}$-face region has a higher piezoresponse magnitude as indicated by contrast, and there is a sharp contrast difference in the piezoresponse phase image, demonstrating inversions in film polarity. (d) Histogram of the PFM phase image (c). The magnitudes of the Ga- and $\mathrm{N}$-face peaks correspond to the relative areas of each region.

magnitude of the total polarization and the corresponding bound surface charge is larger for the $\mathrm{N}$ face $(P$ $=0.0293 \mathrm{C} / \mathrm{m}^{2}, \sigma / e=1.83 \times 10^{13} \mathrm{~cm}^{-2}$ ) in comparison to the Ga-face $\mathrm{GaN} \quad\left(P=-0.0285 \mathrm{C} / \mathrm{m}^{2}, \sigma / e=-1.78\right.$ $\times 10^{13} \mathrm{~cm}^{-2}$ ), which would lead to a higher magnitude of the piezoresponse of the $\mathrm{N}$-face regions. From Fig. 1(b) we also see a higher piezoresponse close to the inversion domain boundaries (IDBs) between $\mathrm{Ga}$ - and $\mathrm{N}$-face regions. It has been suggested ${ }^{18,19}$ that the local strain field increases close to an IDB, possibly explaining the increased piezoresponse at the polarity boundary.

The piezoresponse of the GaN film was found to be 0.6 and $0.3 \mathrm{pm} / \mathrm{V}$ for the $\mathrm{N}$-face and Ga-face regions, respectively. These values are significantly lower than those obtained by macroscopic methods. ${ }^{20,21}$ The relatively low piezoresponse values can be attributed to at least two effects, the first is that the applied field is not uniform over the film and the second is that the displacement will be mechanically constrained by the adjacent nonvibrating regions.

In the PFM phase image [Fig. 1(c)], the $\mathrm{N}$-face regions have a lighter contrast than the Ga-face regions. A histogram representation of the relative areas of the Ga- and N-polarity phases is shown in Fig. 1(d). From this figure it can be seen that while the Ga face vibrates almost in phase with the modulation voltage $\left(\sim 20^{\circ}\right)$, the oscillation of the $\mathrm{N}$ face is out-of-phase with respect to the applied voltage $\left(\sim 160^{\circ}\right)$. In-phase vibration is an indication that the total polarization points toward the substrate, which is consistent with the Gaface polarity. Accordingly, a region with out-of-phase vibration should have polarization directed toward the surface, as in the $\mathrm{N}$-face region. The fact that the phase difference between the Ga- and $\mathrm{N}$-polarity regions is not exactly $180^{\circ}$ 

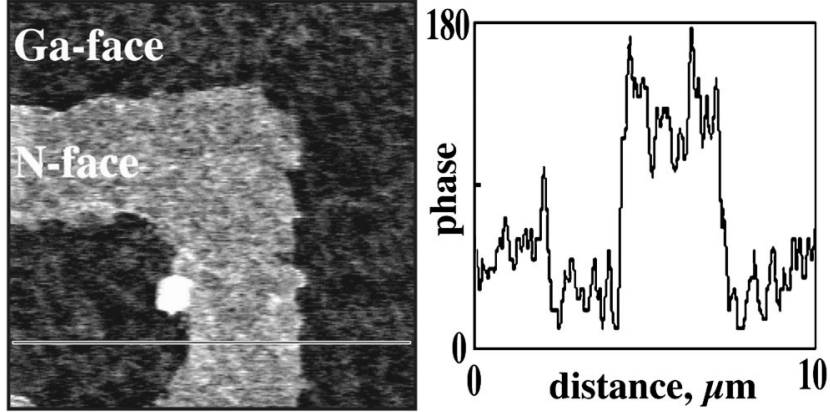

FIG. 2. PFM phase image of Ga- and $\mathrm{N}$-face regions with corresponding cross-sectional profile.

may be attributed to parasitic capacitance of the tip-sample structure.

It should be noted that in the PFM experimental setup both electrostatic forces and the electromechanical response of the surface could contribute to the measured signal. However, diamond coated tips have been shown to be long lasting and ideally suited for PFM since the tip-field electrostatic interaction is minimized when they are used in contact mode due to the high force constant. ${ }^{16}$ In addition, the observed PFM contrast was stable and did not change after subsequent measurements. Images obtained using electrostatic force microscopy were found to be sensitive to the preceding PFM measurements, apparently due to changes in the surface charge distribution which took place during PFM imaging. Therefore, we attribute the observed PFM contrast mainly to the piezoelectric effect. Due to the finite conductivity of the $\mathrm{GaN}$, there will be a redistribution of charge that will partially screen the polarization charge, the effect of which will be explored by investigating the frequency dependence of the piezoresponse. A more complete understanding of the electrostatic and piezoelectric effects, and hence the relationship between such factors as strain and surface charge density, will be studied by comprehensive scanning probe measurements including PFM, electrostatic force microscopy, and scanning Kelvin probe microscopy.

A line scan cross-sectional analysis of the PFM phase image of the GaN heterostructure (Fig. 2) reveals that the lateral resolution achieved in imaging of the $\mathrm{Ga}$ - and $\mathrm{N}$-face regions is about $100 \mathrm{~nm}$. The lateral resolution is limited by the width of the IDB. It is believed that in the case of the sharp IDB the Ga- and $\mathrm{N}$-face regions can be imaged with much higher resolution. ${ }^{11}$

In summary, we have demonstrated the applicability of piezoresponse force microscopy for nanometer scale imaging and piezoelectric measurements of GaN-based lateral polarity heterostructures. The PFM method enables us to identify the location of inversion domain boundaries as well as the polarity of the adjacent GaN crystals and to perform evaluation of the piezoelectric parameters at the nanoscale level.

The authors would like to acknowledge Dr. R. Dimitrov for the realization of $\mathrm{GaN}$ based lateral polarity heterostructures. This work is supported by the Office of Naval Research MURI on Polarization Electronics Contract No. N00014-99-1-0729.

${ }^{1}$ O. Ambacher, J. Majewski, C. Miskys, A. Link, M. Hermann, M. Eickhoff, M. Stutzmann, F. Bernardini, V. Fiorentini, V. Tilak, B. Schaff, and L. F. Eastman, J. Phys.: Condens. Matter 14, 3399 (2002).

${ }^{2}$ A. Zoroddu, F. Bernardini, P. Ruggerone, and V. Fiorentini, Phys. Rev. B 64, 045208 (2001).

${ }^{3}$ O. Ambacher, J. Smart, J. R. Shealy, N. G. Weimann, K. Chu, M. Murphy, W. J. Schaff, L. F. Eastman, R. Dimitrov, L. Wittmer, M. Stutzmann, W. Rieger, and J. Hilsenbeck, J. Appl. Phys. 85, 3222 (1999).

${ }^{4}$ K. V. Smith, E. T. Yu, J. M. Redwing, and K. S. Boutros, J. Electron. Mater. 29, 274 (2000).

${ }^{5}$ G. Koley and M. G. Spencer, J. Appl. Phys. 90, 337 (2001).

${ }^{6}$ K. M. Jones, P. Visconti, F. Yun, A. A. Baski, and H. Morkoc, Appl. Phys. Lett. 78, 2497 (2001).

${ }^{7}$ A. Kholkin, C. Wuetchrich, D. V. Taylor, and N. Setter, Rev. Sci. Instrum. 67, 1935 (1996).

${ }^{8}$ H. Birk, J. Glatz-Reichenbach, Li-Jie, E. Schreck, and K. Dransfield, J. Vac. Sci. Technol. B 9, 1162 (1991).

${ }^{9}$ L. Jie, C. Baur, B. Koslowski, and K. Dransfield, Physica B 204, 318 (1995).

${ }^{10}$ T. Hidaka, T. Maruyama, M. Saitoh, N. Mikoshiba, M. Shimizu, T. Shiosaki, L. A. Wills, R. Hiskes, S. A. Dicarolis, and J. Amano, Appl. Phys. Lett. 68, 2358 (1996).

${ }^{11}$ A. Gruverman, O. Auciello, and H. Tokumoto, Annu. Rev. Mater. Sci. 28, 101 (1998).

${ }^{12}$ S. Hong, E. L. Colla, E. Kim, D. V. Taylor, A. K. Tagantsev, P. Muralt, K. No, and N. Setter, J. Appl. Phys. 86, 607 (1999).

${ }^{13}$ A. Gruverman, O. Auciello, and H. Tokumoto, J. Vac. Sci. Technol. B 14, 602 (1996).

${ }^{14}$ S. V. Kalinin and D. Bonnell, Appl. Phys. Lett. 78, 1116 (2001).

${ }^{15}$ J. A. Christman, S.-H. Kim, H. Maiwa, J.-P. Maria, B. J. Rodriguez, A. I. Kingon, and R. J. Nemanich, J. Appl. Phys. 87, 8031 (2000).

${ }^{16}$ J. A. Christman, R. R. Woolcott, Jr., A. I. Kingon, R. J. Nemanich, Appl. Phys. Lett. 73, 3851 (1998).

${ }^{17}$ R. Dimitrov, V. Tilak, M. Murphy, W. J. Schaff, L. F. Eastman, A. P. Lima, C. Miskys, O. Ambacher, and M. Stutzmann, Mat. Sci. Soc. Symp. 622, T4.6.1 (2000)

${ }^{18}$ J. E. Northrup, Physica B 273-274, 130 (1999).

${ }^{19}$ J. E. Northrup, J. Neugebauer, and L. T. Romano, Phys. Rev. Lett. 77, 103 (1996).

${ }^{20}$ I. L. Guy, S. Muensit, and E. M. Goldys, Appl. Phys. Lett. 75, 4133 (1999).

${ }^{21}$ C. M. Lueng, H. L. W. Chan, C. Surya, and C. L. Choy, J. Appl. Phys. 88, 5360 (2000). 